\title{
Editorial
}

\section{Wir trauern um Hans Westmeyer}

\author{
Olaf Köller
}

Am 5. Juni 2019 ist Hans Westmeyer nicht einmal ein Jahr nach dem Tod von Franz Petermann verstorben. Die Diagnostica hat so in kurzer Zeit zwei der bedeutendsten Herausgeber ihrer Geschichte verloren. Hans Westmeyer war von 1976 bis 1994 Geschäftsführender Herausgeber der Diagnostica und hat die Zeitschrift geprägt, wie kein anderer Herausgeber vor und nach ihm. Mit seinen theoretischen und wissenschaftstheoretischen Beiträgen hat er die Differentielle Psychologie und psychologische Diagnostik über viele Jahre intellektuell bereichert. Ich selbst hatte das Glück Hans Westmeyer, erst nach seiner Zeit bei Diagnostica, im Rahmen der Arbeiten zur Entwicklung von Studienzulassungstests für das Fach Psychologie, kennen- und schätzen zu lernen. Abgesehen von seiner äußerst sympathischen Art hat mich seine besondere Expertise fasziniert und die kurze, aber schöne Kooperation mündete in einem gemeinsamen Artikel in der Psychologischen Rundschau. Die Herausgeberinnen und Herausgeber der Diagnostica verneigen sich vor diesem großartigen Kollegen und werden ihn und seine Arbeiten im Gedächtnis bewahren.

Diagnostica entwickelt sich im Gedenken an Hans Westmeyer weiter. Unsere Zeitschrift ist und bleibt erfolgreich. Gleichwohl - das zeigt zumindest der im letzten Jahr deutlich gesunkene Impact-Factor (2019: 0.488; 2018: 1.074) - sorgt die fortschreitende Internationalisierung der Forschung dafür, dass immer mehr internatio- nale auf Kosten der nationalen Arbeiten zitiert werden. Dies mag damit zusammenhängen, dass internationale Journals es nicht goutieren, wenn Arbeiten in einer anderen Sprache als Englisch zitiert werden. Diagnostica ist mit diesem Problem nicht allein, vielmehr betrifft das deutliche Absinken des Impact Factors im Journal Citation Report 2020 fast alle deutschsprachigen psychologischen Zeitschriften. Wir sehen diese Verlagerung zugunsten internationaler Zeitschriften allerdings nicht bei der Zahl der Einreichungen in Diagnostica. Diese bleibt sehr hoch, entsprechend ist die Zahl der Manuskripte, die in der ersten Begutachtungsrunde akzeptiert wurden, nach wie vor sehr gering (deutlich unter $20 \%$ ) und signalisiert die hohen Qualitätsstandards, die wir an Beiträge anlegen. Dies mag weiterhin Ansporn für die Autorinnen und $\mathrm{Au}-$ toren sein, ihre besten deutschsprachigen Arbeiten im Bereich der Diagnostischen und Differenziellen Psychologie bei Diagnostica einzureichen.

Abschließend gilt mein großer Dank dem Redaktionsteam in Kiel, das die Einreichungen mit großer Akribie begleitet und dafür sorgt, dass die ganz große Mehrzahl der Einreichungen zügig betreut und zu einer Entscheidung über Annahme oder Ablehnung gebracht wird.

Olaf Köller

Geschäftsführender Herausgeber 